Check for updates

Cite this: Mater. Adv., 2021, 2,6403

Received 19th May 2021 Accepted 22nd August 2021

DOI: 10.1039/d1ma00450f

rsc.li/materials-advances

\title{
A XANES study of lithium polysulfide solids: a first-principles study $\dagger$
}

\author{
Qing Guo, (D) *ab Kah Chun Lau (D) *b and Ravindra Pandey (D) ${ }^{\mathrm{a}}$
}

\begin{abstract}
X-ray absorption spectroscopy (XAS) is used for capturing the reaction mechanisms at the molecular level via the determination of local electronic configurations inside Li-S batteries. In this paper, a comprehensive investigation of the S K-edge X-ray absorption near-edge structure (XANES) of a series of stoichiometric lithium polysulfide solids was performed, assuming that these polysulfide solids are formed during the battery cycling process. The results based on density functional theory show that the pre-edge peak in the $\mathrm{S}$ K-edge XANES spectra is not a common feature in the solid phase in contrast to the case of pristine polysulfide molecules where the pre-edge peak constitutes a unique signature when dissolved in liquid electrolytes. A general redshift in the S K-edge XANES peak is found when a Li vacancy is introduced in these lithium polysulfide solids. Furthermore, the first peak positions of the $\mathrm{S} K$-edge XANES spectra of the lithium polysulfide solids appear within a small range (2471-2473 eV) overlapping with the white line of the $\alpha-S_{8}$ bulk ( $\left.2472 \mathrm{eV}\right)$. Therefore, the results suggest that the use of additional characterization methods might be necessary for accurately identifying the elusive solid phase lithium polysulfides in Li-S batteries instead of relying only on S K-edge XANES spectroscopy.
\end{abstract}

\section{Introduction}

The need for renewable energy sources alternative to fossil fuels has become urgent due to the increasing environmental pressure. To reduce greenhouse gas emissions, full electric vehicles (EVs) powered by Li-ion batteries (LIBs) are becoming an important alternative to the traditional internal combustion engine vehicles. However, a higher energy density battery is still needed to extend the cruising range of EVs at a lower cost. The Li-S battery system is an attractive candidate to substitute LIBs because of its ultrahigh theoretical specific energy (2500 $\left.\mathrm{W} \mathrm{h} \mathrm{kg}^{-1}\right)$, which is a few times higher than that of the current LIBs. ${ }^{1-4}$ A major obstacle that prevents the commercialization of $\mathrm{Li}-\mathrm{S}$ batteries is the "shuttle effect", a phenomenon associated with the high solubility of intermediate discharge products (polysulfides) in liquid electrolytes which will reduce the active sulfur and corrode the lithium metal..$^{5-7}$ Several attempts have been made to suppress the shuttle effect. One effective way is trapping intermediate polysulfides to confine them within the electrodes with which only $\mathrm{Li}^{+}$can diffuse through the boundary, such as microporous structures $^{8-11}$ and polymer coatings. ${ }^{7,12}$ The spatial confinement

\footnotetext{
${ }^{a}$ Department of Physics, Michigan Technological University, Houghton, MI 49931, USA. E-mail: qinguo@mtu.edu

${ }^{b}$ Department of Physics and Astronomy, California State University, Northridge, CA 91330, USA. E-mail: kahchun.lau@csun.edu

$\dagger$ Electronic supplementary information (ESI) available. See DOI: 10.1039/ d1ma00450f
}

may lead to the formation of solid phase discharge products, and various stoichiometric ratios of $\mathrm{Li}$ and $\mathrm{S}\left(\mathrm{Li}_{x} \mathrm{~S}_{y}\right)$ will vary successively from elemental sulfur to intermediate polysulfides and subsequently to the final product $\mathrm{Li}_{2} \mathrm{~S}$ when the battery is discharged continuously. A recent experimental study demonstrated a quasi-solid state sulfur conversion during the Li-S battery discharging process, which also indicates the presence of solid phase lithium polysulfide intermediates. ${ }^{13}$

To understand these elusive intermediate species, sulfur K-edge X-ray absorption near edge structure (XANES) is a promising characterization technique to probe the sulfur element's local chemical environment/oxidation state, which has been widely used for performing a comprehensive study of the reaction mechanism inside Li-S batteries. ${ }^{7,10,14-17}$ However, interpreting the S K-edge XANES results is quite problematic due to a great variety of metastable polysulfide intermediates and the lack of XANES spectral reference fingerprints, especially in solid phase lithium polysulfides. In contrast to the lithium polysulfide molecules dissolved in liquid electrolytes, single crystalline phase $\mathrm{Li}_{2} \mathrm{~S}_{x}$ are barely synthesized experimentally due to the thermodynamically meta-stable ${ }^{18}$ and complex properties of these lithium-sulfur compounds. Experimentally, mixtures with a specific stoichiometric ratio of lithium sulfide and sulfur have been used to establish the standards of $\mathrm{Li}_{2} \mathrm{~S}_{x} .{ }^{10,15}$ The pre-edge peak around $2470 \mathrm{eV}$ of the $\mathrm{S}$ K-edge XANES spectrum is considered as the signal of linear $\mathrm{S}_{x}{ }^{2-}$ dianion formation. ${ }^{10,16,19}$ To aid the XANES spectra in experiments, first-principles-based calculations have been performed for a single polysulfide $\left(\mathrm{Li}_{2} \mathrm{~S}_{x}\right)$ molecule dissolved in tetraglyme (TEDME) 
electrolyte by Pascal et $a .^{20}$ The pre-edge and main peaks are assigned to the terminal and internal $\mathrm{S}$ atoms of the linear polysulfide dianions, respectively. Compared to polysulfide molecules in liquid electrolytes, fewer studies have focused on the solid phase of lithium polysulfides due to their low stability under ambient conditions. Recently, an experimental XANES study suggested that the solvation of polysulfides can be well suppressed by the combination of an alucone-coated $\mathrm{C}-\mathrm{S}$ cathode and a carbonate-based electrolyte. ${ }^{7}$ A solid phase transition mechanism from sulfur directly to $\mathrm{Li}_{2} \mathrm{~S}$ was proposed as evidenced by the absence of the pre-edge peak of polysulfides in the spectra. ${ }^{7}$ Since there is a lack of a systematic study of XANES spectra for a wide range of solid phase stoichiometric lithium polysulfides, the above interpretation appears to be somewhat ambiguous.

In this paper, we have investigated the XANES spectra of lithium polysulfide crystalline structures ranging from $\mathrm{Li}_{2} \mathrm{~S}_{2}$ to $\mathrm{Li}_{2} \mathrm{~S}_{8}$. The predicted structures are thermodynamically stable and are metallic or semiconducting with a small band gap compared to that of the insulating $\mathrm{Li}_{2} \mathrm{~S} .{ }^{18}$ Besides, the unique molecular signatures of $S_{x}$ chains in the solid phase polysulfides are well preserved, analogous to conventional molecular solids. In contrast to the XANES spectra of pristine polysulfide $\left(\mathrm{S}_{x}{ }^{2-}\right)$ molecules in solution, the iconic pre-edge peak in the $\mathrm{S}$ K-edge XANES spectra of $\mathrm{S}_{x}{ }^{2-}$ is predicted to be absent in solids. Due to the significant effect of a $\mathrm{Li}$ vacancy on the XANES spectra, the results show that the local coordination of sulfur species is an important factor in dictating the XANES characteristics of lithium polysulfides.

\section{Computational method}

The search for low-energy crystalline structures of lithium polysulfides $\left(\mathrm{Li}_{2} \mathrm{~S}_{x}, x=3-8\right)$ was performed with the CALYPSO code using the particle swarm optimization methodology. ${ }^{21,22}$ The validity of this methodology for finding stable or metastable structures has been confirmed in a variety of systems, including the Li-S system..$^{23-25}$ The structures totaling around $\approx 900$ consisting of two $\mathrm{Li}_{2} \mathrm{~S}_{x}$ formula units were searched separately for each $\mathrm{Li}_{2} \mathrm{~S}_{x}$ stoichiometry. The underlying geometry optimization and electronic property calculations were carried out in the framework of density functional theory (DFT) using the VASP code. ${ }^{26,27}$ The projector augmented wave (PAW) method ${ }^{28}$ was applied using generalized gradient approximation (GGA) with the Perdew-Burke-Ernzerhof (PBE) exchange-correlation functional. ${ }^{29}$ Grimme's D2 method $^{30}$ was applied to represent van der Waals interactions. ${ }^{31,32}$ Throughout this work, an energy cutoff of $500 \mathrm{eV}$ and the MonkhorstPack $K$-point meshes with the spacing values of $0.5 \AA^{-1}$ and $0.2 \AA^{-1}$ in the reciprocal space were used for geometry optimization and static calculation, respectively. During the geometry optimization, the residual force on each atom was less than $0.01 \mathrm{eV} \AA^{-1}$, while the convergence criterion of total energy for the static calculation was set to be $10^{-7} \mathrm{eV}$.

Theoretical calculations for X-ray absorption spectra (XAS) were performed within the excited-state core hole $(\mathrm{XCH})$ approach $^{33}$ with the modified sulfur pseudopotential with a core hole at the 1s level using the Quantum-ESPRESSO (QE) package. ${ }^{34,35}$ To obtain the wave function of the excited system, a self-consistent field (SCF) calculation on lithium polysulfides was carried out using the PWscf code ${ }^{34}$ within the QE package. Subsequently, the XANES spectra were computed via a continued fraction approach using the XSpectra code. ${ }^{36,37}$ The structural configurations of lithium polysulfides were obtained at the same level of theory using VASP. Furthermore, the energy alignment scheme proposed by Prendergast et al. was adopted, ${ }^{20,38,39}$ which allows the comparison of the DFT results with the experimental XAS results. Following this scheme, we apply the energy shift that was obtained by matching the calculated and experimental XAS spectra of an isolated $\mathrm{S}_{2}$ molecule ${ }^{40}$ to the $S$ K-edge XANES spectra of lithium polysulfides. The validity of this approach has previously been confirmed, ${ }^{14-16}$ including examples of elemental sulfur and crystalline $\mathrm{Li}_{2} \mathrm{~S}$.

\section{Results and discussion}

In the following, three low-energy structural configurations consisting of diverse sulfur chain (clusters) species for each $\mathrm{Li}_{2} \mathrm{~S}_{x}(x=2-8)$ (labeled as str-1, str-2, and str-3) in a crystal cell are chosen in the discussion instead of an exhaustive study of all possible configurations. It is to be noted that these polysulfides' solid phases were found to be metastable based on the formation energy convex hull relative to that of $\mathrm{Li}_{2} \mathrm{~S}$ under ambient conditions. ${ }^{18}$ For each low-energy crystalline structure, we found that the $\mathrm{Li}_{2} \mathrm{~S}_{x}$ stoichiometry, in general, does not guarantee the presence of equivalent sulfur chains $\left(\mathrm{S}_{x}\right)$ or polysulfide $\left(\mathrm{Li}_{2} \mathrm{~S}_{x}\right)$ species in the predicted structural configuration. In other words, various sulfur chains or polysulfide species can be present in any pre-determined chemical stoichiometry of the crystalline solid as a local minimum during the search for low-energy crystalline structures of lithium polysulfides in DFT calculations. This unique trend is found to be drastically different compared to pristine stoichiometric lithium polysulfide molecules. For example, the lowest energy $\mathrm{Li}_{2} \mathrm{~S}_{4}-1$ structural configuration prefers the presence of $\mathrm{S}_{3}$ and $\mathrm{S}_{5}$ chains over the uniform $\mathrm{S}_{4}$ chains in the lattice. A similar trend was also observed in the molecular dynamics simulations of Li-S nanoparticles. ${ }^{42}$ From our recent findings, ${ }^{18}$ the studied lithium polysulfides are only $22-127 \mathrm{meV}$ per atom unfavorable compared to the stable $\alpha$-sulfur and lithium sulfide. This energy range is comparable to those of many metastable solids in Inorganic Crystal Structure Database (ICSD), ${ }^{43}$ indicating that the lithium polysulfide solids may possibly be synthesized experimentally. However, the similarity in cohesive energy values also indicates that these lithium polysulfide solid phases may co-exist or in an amorphous phase ${ }^{13}$ in experiments.

The projected electronic density of states (PDOS) (Fig. S1, $\mathrm{ESI} \dagger$ ) shows that the electronic states are solely attributed to sulfur $3 p$ orbitals near the Fermi level. On the other hand, the analysis of Bader's charges found that the terminal sulfur $\left(\mathrm{S}_{\mathrm{T}}\right)$ 

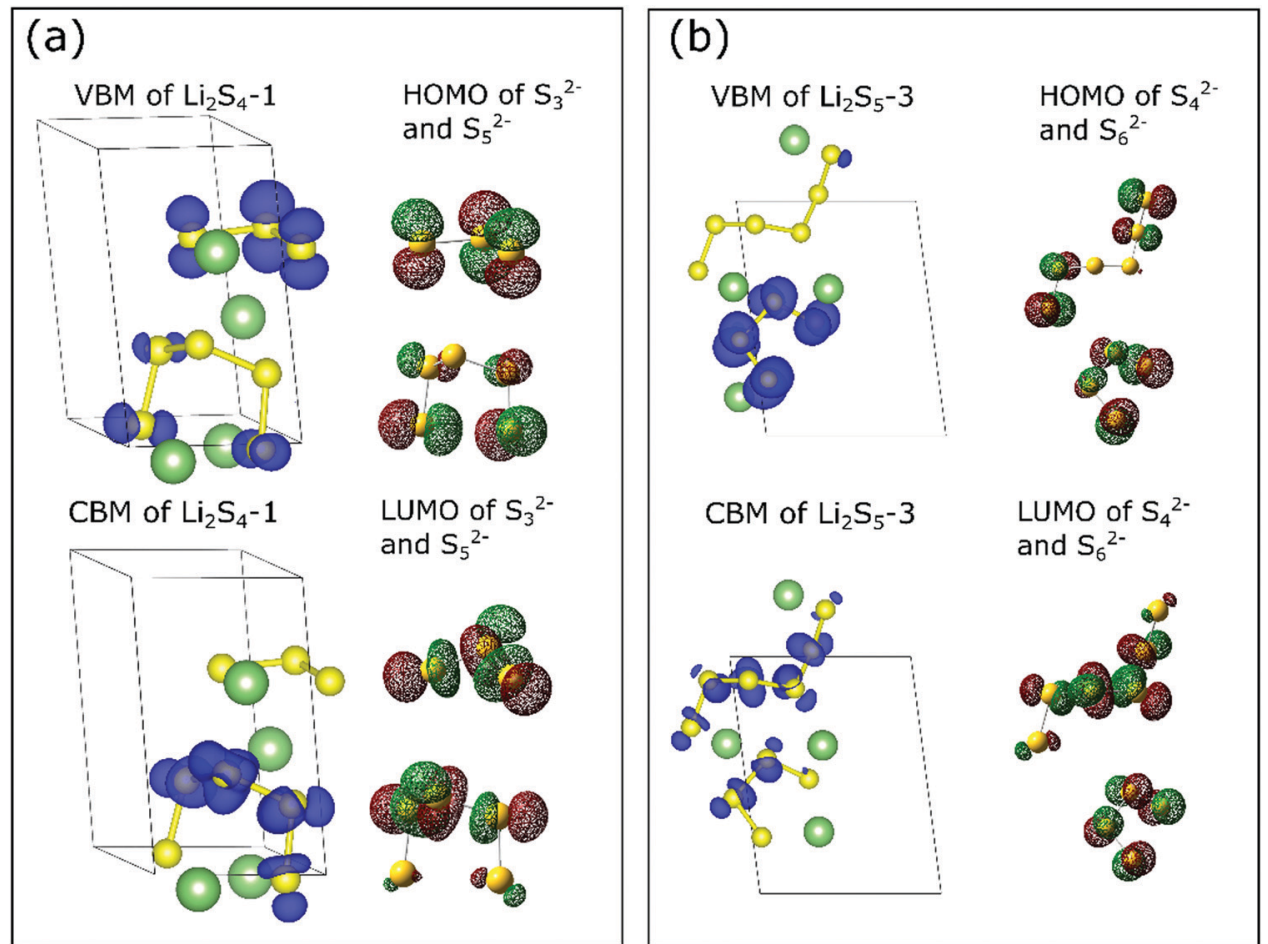

Fig. 1 Projected charge distribution of the valence band maximum (VBM) and the conduction band minimum (CBM) of (a) $\mathrm{Li}_{2} \mathrm{~S}_{4}-1$ and (b) $\mathrm{Li}_{2} \mathrm{~S}_{5}-3$ solids and the HOMO and LUMO of the pristine sulfur chain dianions ((a) $S_{3}{ }^{2-} \& S_{5}{ }^{2-}$ and (b) $S_{4}{ }^{2-} \& S_{6}{ }^{2-}$ ).

atoms possess higher charges than the internal sulfur bridge $\left(\mathrm{S}_{\mathrm{B}}\right)$ atoms in a linear sulfur chain (e.g., $\mathrm{S}_{3} / \mathrm{S}_{5}$ in $\mathrm{Li}_{2} \mathrm{~S}_{4}-1, \mathrm{~S}_{4} / \mathrm{S}_{6}$ in $\mathrm{Li}_{2} \mathrm{~S}_{5}-3$ and $\mathrm{S}_{8}$ in $\mathrm{Li}_{2} \mathrm{~S}_{8}-1$ ), and therefore they are likely to form a $\mathrm{S}_{x}{ }^{2-}$ dianion, which is analogous to the recently reported lithium polysulfide molecule that is dissolved in a liquid electrolyte $^{20}$ (see Tables S1 and S2, ESI $\dagger$ ).

To highlight the unique features of sulfur chains in the lithium polysulfide solids, we now plot the projected charge density associated with the valence and conduction bands and compare it with the molecular orbitals of the corresponding isolated polysulfide dianions $\left(\mathrm{S}_{x}{ }^{2-}\right) .{ }^{44}$ For the sake of brevity, only structures $\mathrm{Li}_{2} \mathrm{~S}_{4}-1$ and $\mathrm{Li}_{2} \mathrm{~S}_{5}-3$ are discussed as they consist of $S_{3} / S_{5}$ and $S_{4} / S_{6}$ chains. The unique charge distributions of $\mathrm{Li}_{2} \mathrm{~S}_{4}-1$ and $\mathrm{Li}_{2} \mathrm{~S}_{5}-3$ can be clearly observed in Fig. 1 . We found that the iso-surface of $\mathrm{Li}_{2} \mathrm{~S}_{4}-1$ exhibits very similar features to the highest occupied molecular orbital (HOMO) and the lowest unoccupied molecular orbital (LUMO) of the isolated molecular dianion $\left(\mathrm{S}_{x}{ }^{2-}\right)$ counterpart. For example, the projected charge density at the highest occupied valence band (Fig. 1a-top) of $\mathrm{Li}_{2} \mathrm{~S}_{4}-1$ is similar to those at the HOMOs of both pristine $\mathrm{S}_{3}{ }^{2-}$ and $\mathrm{S}_{5}{ }^{2-}$ molecules, whereas the value at the lowest unoccupied conduction band (Fig. 1a-bottom) is similar to that at the LUMO of the pristine $\mathrm{S}_{5}{ }^{2-}$ molecule. For the $\mathrm{Li}_{2} \mathrm{~S}_{4}-1$ solid, this similarity suggests that the HOMOs of the constituent $\mathrm{S}_{3}{ }^{2-}$ and $\mathrm{S}_{5}{ }^{2-}$ species are associated with the same energy level near the Fermi level. On the other hand, the LUMO of $\mathrm{S}_{3}{ }^{2-}$ is higher than that of $\mathrm{S}_{5}{ }^{2-}$ in orbital energy. Later, we show that this unique feature is reflected in its XANES spectrum. Meanwhile, for the $\mathrm{Li}_{2} \mathrm{~S}_{5}-3$ structure (Fig. 1b), the non-degenerate HOMO and degenerate LUMO levels are associated with the $S_{4}$ and $S_{6}$ chains. In both cases (i.e., $\mathrm{Li}_{2} \mathrm{~S}_{4}-1$ and $\mathrm{Li}_{2} \mathrm{~S}_{5}-3$ ), the similarity in the electronic charge density of crystalline polysulfides to that of the polysulfide dianions suggests that the unique orbital characteristics of molecular polysulfides are generally wellpreserved in the solid phase. This intriguing finding implies that the lithium polysulfide solids are more like molecular crystals, rather than ionic crystals, and therefore the van der Waals interaction terms governing the stability of these compounds have also been highlighted recently. ${ }^{31,32,41}$

As highlighted in the previous discussion, the individual molecular features of $\mathrm{S}_{x}{ }^{2-}$ anions are generally well preserved in the lithium polysulfide solids. Thus, a comparative study of the XANES spectra relative to the dissolved lithium polysulfide molecules is important. In the case of the dissolved polysulfide molecules, the pre-edge XANES peak of $S_{x}{ }^{2-}$ has been attributed to different XANES peak positions from the terminal and internal $S$ atoms in a polysulfide chain. ${ }^{20}$ In the present case of lithium polysulfide solids, despite having similar charge features in both terminal and internal $S$ atoms, no distinguishable pre-edge XANES peak around $2470 \mathrm{eV}$ (i.e., S K-edge) is predicted, except the case of $\mathrm{Li}_{2} \mathrm{~S}_{6}-1$ (Fig. 2). For this crystalline structure $\left(\mathrm{Li}_{2} \mathrm{~S}_{6}-1\right)$, this configuration is unique and only $\mathrm{S}_{2}$ dimers are present in crystalline lattices, in which the nearest two parallel $\mathrm{S}_{2}$ dimers are merely separated by $2.67 \AA$. This small distance induces the molecular orbital split between $\pi_{3 \mathrm{px}}{ }^{*}$ and $\pi_{3 \mathrm{py}}{ }^{*}$ of a sulfur dimer which represents the final states for the $\mathrm{S} 1 \mathrm{~s}$ electron excitation. From our analysis, the pre-edge feature of $S$ K-edge in XANES spectra for $\mathrm{Li}_{2} \mathrm{~S}_{6}-1$ 

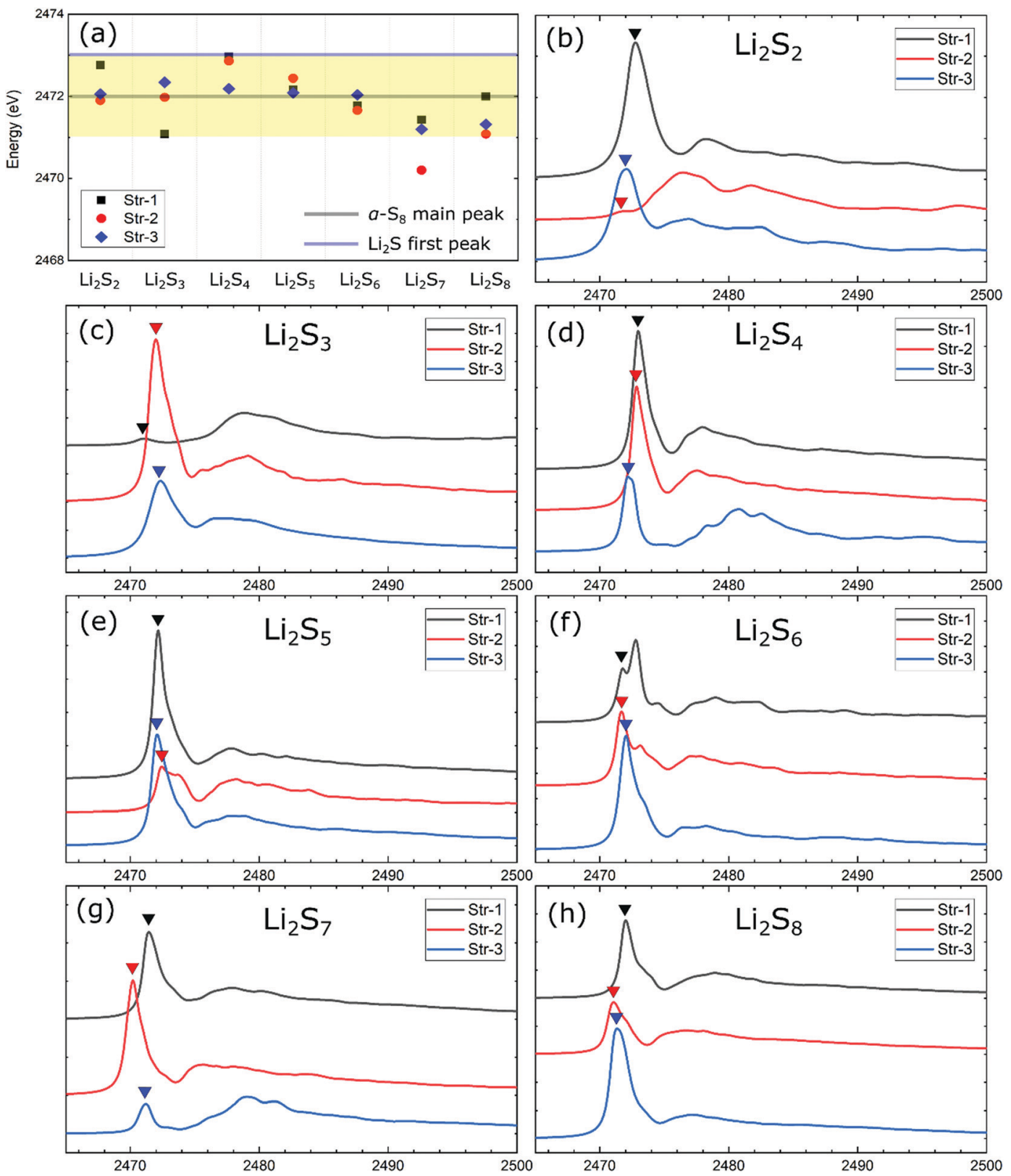

Fig. 2 Calculated XANES spectra of $\mathrm{Li}_{2} \mathrm{~S}_{x}(x=2-8)$ solids. (a) The first peak positions for all the $\mathrm{Li}_{2} \mathrm{~S}_{x}$ structures' XANES spectra. The yellow region represents the energy range of 2471-2473 eV. The XANES spectrum for each structure with a stoichiometry of (b) $\mathrm{Li}_{2} \mathrm{~S}_{2}$, (c) $\mathrm{Li}_{2} \mathrm{~S}_{3},(\mathrm{~d}) \mathrm{Li}_{2} \mathrm{~S}_{4}$, (e) $\mathrm{Li}_{2} \mathrm{~S}_{5}$, (f) $\mathrm{Li}_{2} \mathrm{~S}_{6},(\mathrm{~g}) \mathrm{Li}_{2} \mathrm{~S}_{7}$, and (h) $\mathrm{Li}_{2} \mathrm{~S}_{8}$. The solid triangles indicate the first peak positions for each spectrum.

(Fig. 2f) is attributed to this molecular orbital split as shown in Fig. S2 in the ESI. $\dagger$

We note that the absence of the pre-edge XANES peak was reported in carbonate-based electrolyte Li-S batteries and was attributed to the insolubility or low solubility of polysulfides in carbonate-based electrolytes. ${ }^{7,45}$ In our opinion, the lack of distinguishable pre-edge XANES characteristics of polysulfide chains is due to the distinctive bonding environment of the polysulfide molecules in the solid phase. Instead of being solvated by organic molecules in liquid electrolytes, polysulfides in the solid phase are exposed to more substantive intermolecular interactions with the surrounding sulfur atoms including Li-ions due to the periodicity of the crystalline solid.
Considering that there exist several inequivalent $\mathrm{S}$ atoms in most of the lithium polysulfide structures, an exhaustive discussion on their XAS spectra could be made easier by focusing on the first adsorption peak position of the $\mathrm{S}$ K-edge in XANES spectra. Fig. 2 displays the predicted XANES spectra for each stoichiometric lithium polysulfide solids from $\mathrm{Li}_{2} \mathrm{~S}_{2}$ to $\mathrm{Li}_{2} \mathrm{~S}_{8}$. Unlike the pristine polysulfide molecules that dissolve in etherbased electrolytes, ${ }^{20}$ we found that the signature feature of the pre-edge peak is missing in the polysulfide solids. The analysis of the XANES spectra of individual atoms in the same chains found no specific differences between the terminal and the bridge $\mathrm{S}$ atoms (Fig. S3, ESI $\dagger$ ). The first S K-edge peaks of these $\operatorname{Li}_{2} \mathrm{~S}_{x}(x=2-8)$ solids are located within a proximity of 
$\sim 2471 \mathrm{eV}-2473 \mathrm{eV}$ (Fig. 2a), and overlaps with the first peak of $\alpha-\mathrm{S}_{8}$ at $2472 \mathrm{eV}$, but are clearly distinguishable from those of the $\mathrm{S}^{2-}$ ionic species of $\mathrm{Li}_{2} \mathrm{~S}$ solids that are located around $2473 \mathrm{eV}$ and $2475 \mathrm{eV}$. ${ }^{46}$ Therefore, in contrast to the individual polysulfide molecules which dissolved in ether-based electrolytes, ${ }^{10,47}$ the results for polysulfide solids suggest that a distinction between polysulfide solids and the sulfur bulk solely based on the first adsorption peak position of the S K-edge in XANES spectra can be ambiguous or less conclusive.

We now focus on the analysis of XANES spectra obtained for the representative $\mathrm{Li}_{2} \mathrm{~S}_{4}-1$ and $\mathrm{Li}_{2} \mathrm{~S}_{5}-3$ configurations consisting of a range of sulfur chains from $\mathrm{S}_{3}$ to $\mathrm{S}_{6}$ in the crystal lattice. For $\mathrm{Li}_{2} \mathrm{~S}_{4}-1$, the XANES main peak located at $\sim 2473.0 \mathrm{eV}$ is mainly dominated by the contribution of the $S_{5}$ chain. For the $S_{3}$ component, the associated peak is located slightly higher at $\sim 2473.2 \mathrm{eV}$ with a much lower intensity (Fig. 3a). Interestingly, this is consistent with the projected electronic density of states, which highlights the dominant contribution of $S_{5}$ to forming the conduction bands (Fig. S6, ESI $\dagger$ ). For $\mathrm{Li}_{2} \mathrm{~S}_{5}-3$, the XANES main peak is located at $\sim 2472.1 \mathrm{eV}$. However, in contrast to $\mathrm{Li}_{2} \mathrm{~S}_{4}-1$, the XANES main peak of $\mathrm{Li}_{2} \mathrm{~S}_{5}-3$ is found to be equally contributed by $S_{4}$ and $S_{6}$ chains (Fig. $3 b$ ). This equivalency is reflected in the degenerate lowest unoccupied bands that are attributed to both $S_{4}$ and $S_{6}$ chains (Fig. 1b), providing the $S 3 p$ electronic states for the $\mathrm{S} 1 \mathrm{~s}$ electron excitation. As expected, the electronic density of states affirms the contributions of both $\mathrm{S}_{4}$ and $\mathrm{S}_{6}$ chains in composing the lower conduction bands (Fig. S6, ESI $\dagger$ ).

Next, we determine the characteristic features of XANES spectra of non-stoichiometric $\mathrm{Li}_{2-y} \mathrm{~S}_{x}(y=0.5,1$, and 1.5) solids which are particularly relevant to the delithiation process during the charging process of the $\mathrm{Li}-\mathrm{S}$ battery. Considering the representative $\mathrm{Li}_{2} \mathrm{~S}_{4}-1$ and $\mathrm{Li}_{2} \mathrm{~S}_{8}-1$ structural configurations,

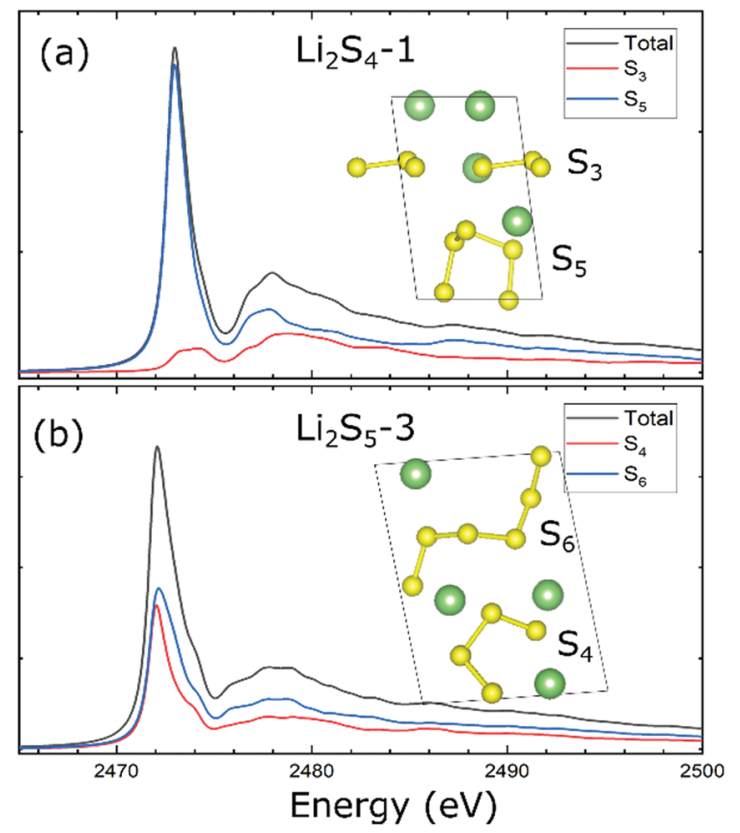

Fig. 3 Calculated XANES spectra of (a) $\mathrm{Li}_{2} \mathrm{~S}_{4}-1$ and (b) $\mathrm{Li}_{2} \mathrm{~S}_{5}-3$ configurations. one, two, or three $\mathrm{Li}$ vacancies were created in the crystal lattice which leads to $\mathrm{Li}_{1.5} \mathrm{~S}_{4}, \mathrm{Li}_{1} \mathrm{~S}_{4}, \mathrm{Li}_{0.5} \mathrm{~S}_{4}$, and $\mathrm{Li}_{1} \mathrm{~S}_{8}$ structural configurations as shown in Fig. $4 \mathrm{a}$ and c. Subsequently, the optimized non-stoichiometric configurations were used to determine their XANES spectra as shown in Fig. 4b and d.

As shown in Fig. 4 (Fig. S4, ESI $\dagger$ ), a Li vacancy (i.e., $\mathrm{Li}_{1.5} \mathrm{~S}_{4}$ ) in the stoichiometric $\mathrm{Li}_{2} \mathrm{~S}_{4}$ induces an energy redshift of $1.0 \mathrm{eV}$ in the XANES K-edge peak which can be attributed to the lowering of the Fermi level through a missing $\mathrm{Li}$ atom in the unit cell. This is followed by the second $\mathrm{Li}$ vacancy (i.e., $\mathrm{Li}_{1} \mathrm{~S}_{4}$ ) and induction of a pre-edge-like feature in the $S$ K-edge XANES spectra. For the non-stoichiometric $\mathrm{Li}_{1} \mathrm{~S}_{4}$, the XANES peak is associated with the total charge imbalance between $\mathrm{S}_{3}\left(0.44 \mathrm{e}^{-}\right)$ and $S_{5}\left(1.29 \mathrm{e}^{-}\right)$chains in the crystalline lattice due to the presence of $\mathrm{Li}$ vacancies. As shown in Fig. S5 (ESI $\dagger$ ), the $\mathrm{S}_{3}$ unit is lowered $\sim 1.2 \mathrm{eV}$ relative to that associated with an $S_{5}$ unit, and this induces a pre-edge-like feature (Fig. 4b). More $\mathrm{Li}$ vacancies (i.e., $3 \mathrm{Li}$ vacancies, $\mathrm{Li}_{0.5} \mathrm{~S}_{4}$ ) further lowered the energy level of the pre-edge peak and caused a redshift (green line) as shown in Fig. 4b. Therefore, one can conclude that the pre-edge feature in the S K-edge spectra can be associated with the peak divergence for different $\mathrm{S}$ chains in the stoichiometric $\mathrm{Li}_{2} \mathrm{~S}_{x}$ solids which is induced by the presence of Li vacancies (e.g., $\mathrm{Li}_{2} \mathrm{~S}_{4}-1$ ) during the delithiation process. As noted, the origin of this pre-edge feature in polysulfide solids is quite different from that attributed to the S-terminal species found in the polysulfide molecules ${ }^{20}$ dissolved in liquid electrolytes.

In contrast to $\mathrm{Li}_{2} \mathrm{~S}_{4}-1$, the effect of $\mathrm{Li}$ vacancy-induced changes in the $\mathrm{S}$ K-edge spectra of $\mathrm{Li}_{2} \mathrm{~S}_{8}-1$ is found to be less significant during the delithiation process, which may be due to a smaller $\mathrm{Li} / \mathrm{S}$ ratio in its unit cell. For example, the redshift of the XANES peak is only $\sim 0.75 \mathrm{eV}$ and is found to be independent or insensitive to the position of the Li vacancy (Fig. 4c and d) in the non-stoichiometric $\mathrm{Li}_{1} \mathrm{~S}_{8}$. Interestingly, the removal of two $\mathrm{Li}$ atoms from a $\mathrm{Li}_{2} \mathrm{~S}_{8}$ chain (Fig. 4c) facilitates the formation of an $S_{8}$ ring in an equilibrium configuration, thereby confirming the fact that opening/closing of the $\mathrm{S}_{8}$ ring is a reversal reaction ${ }^{48}$ depending on the lithiation/delithiation process in the $\mathrm{Li}-\mathrm{S}$ battery system.

\section{Summary}

In this paper, the XANES spectra of a series of lithium polysulfide solids ranging from $\mathrm{Li}_{2} \mathrm{~S}_{2}$ to $\mathrm{Li}_{2} \mathrm{~S}_{8}$ were calculated. The results based on DFT calculations show distinctive molecular features suggesting the lithium polysulfide solids to be more like molecular solids. Analyses of the molecular orbitals, DOS near the Fermi level, and Bader charge distribution facilitate the assignment of the first peaks of the S K-edge spectra in polysulfide solids. In contrast to the pristine lithium polysulfide molecules dissolved in liquid electrolytes, the pre-edge peak is not found to be the common feature among the stoichiometric polysulfide solids, although the non-stoichiometric solids may exhibit the pre-edge feature in the $\mathrm{S}$ K-edge XANES spectra through the presence of the Li vacancy. Compared to stoichiometric lithium 

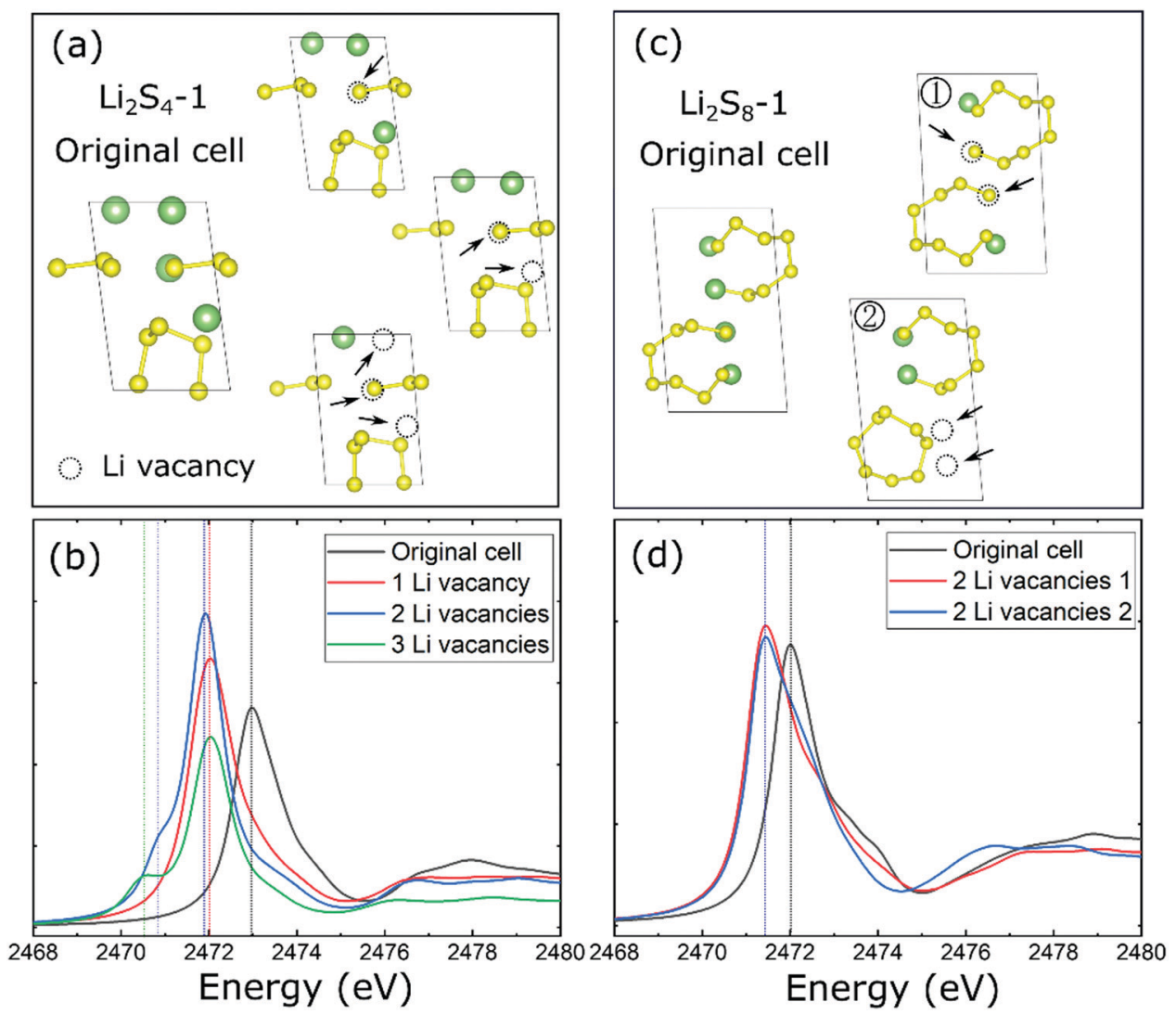

Fig. 4 (a) The structures of $\mathrm{Li}_{2} \mathrm{~S}_{4}-1$ with/without vacancies and (b) their calculated XANES spectra. (c) The structures of $\mathrm{Li}_{2} \mathrm{~S}_{8}-1$ with/without vacancies and (d) their calculated XANES spectra.

polysulfide solids, the redshift of the XANES peaks is commonly found in non-stochiometric solids when a Li vacancy is introduced. For these polysulfide solids, the first peak positions of the $S$ K-edge XANES spectra are located within a small range of 2471-2473 eV which strongly overlaps with that of the $\alpha-S_{8}$ bulk at $\sim 2472 \mathrm{eV}$ but it was found to be different from that of the $\mathrm{S}^{2-}$ species in $\mathrm{Li}_{2} \mathrm{~S}$ which is located at $\sim 2473-2475 \mathrm{eV}$. Overall, it is, therefore, essential to use complementary materials characterization techniques such as IR spectroscopy, Raman spectroscopy, or a "multi-modal" approach, ${ }^{49}$ in addition to XANES spectroscopy, to fully characterize the presence of polysulfides in the $\mathrm{Li}-\mathrm{S}$ battery system.

\section{Conflicts of interest}

There are no conflicts of interest to declare.

\section{Acknowledgements}

The superior, high-performance computing cluster at Michigan Technological University was used to perform electronic structure calculations in this paper. Helpful support from Dr S Gowtham is gratefully acknowledged. K.C. Lau acknowledges the faculty start-up funding provided by California State University, Northridge.

\section{References}

1 P. G. Bruce, S. A. Freunberger, L. J. Hardwick and J.-M. Tarascon, $\mathrm{Li}-\mathrm{O}_{2}$ and $\mathrm{Li}-\mathrm{S}$ Batteries with High Energy Storage, Nat. Mater., 2012, 11, 19.

2 D. Peramunage and S. Licht, A Solid Sulfur Cathode for Aqueous Batteries, Science, 1993, 261, 1029-1032.

3 A. Fotouhi, D. J. Auger, L. O'Neill, T. Cleaver and S. Walus, Lithium-Sulfur Battery Technology Readiness and Applications-a Review, Energies, 2017, 10, 1937.

4 S. Evers and L. F. Nazar, New Approaches for High Energy Density Lithium-Sulfur Battery Cathodes, Acc. Chem. Res., 2013, 46, 1135-1143.

5 Y. V. Mikhaylik and J. R. Akridge, Polysulfide Shuttle Study in the Li/S Battery System, J. Electrochem. Soc., 2004, 151, A1969-A1976.

6 Y. X. Yin, S. Xin, Y. G. Guo and L. J. Wan, Lithium-Sulfur Batteries: Electrochemistry, Materials, and Prospects, Angew. Chem., Int. Ed., 2013, 52, 13186-13200.

7 X. Li, M. Banis, A. Lushington, X. Yang, Q. Sun, Y. Zhao, C. Liu, Q. Li, B. Wang and W. Xiao, A High-Energy Sulfur Cathode in Carbonate Electrolyte by Eliminating Polysulfides Via Solid-Phase Lithium-Sulfur Transformation, Nat. Commun., 2018, 9, 1-10.

8 B. Zhang, X. Qin, G. Li and X. Gao, Enhancement of Long Stability of Sulfur Cathode by Encapsulating Sulfur into 
Micropores of Carbon Spheres, Energy Environ. Sci., 2010, 3, 1531-1537.

9 W. Zhou, Y. Yu, H. Chen, F. J. DiSalvo and H. c. D. Abruña, Yolk-Shell Structure of Polyaniline-Coated Sulfur for Lithium-Sulfur Batteries, J. Am. Chem. Soc., 2013, 135, 16736-16743.

10 M. Cuisinier, P.-E. Cabelguen, S. Evers, G. He, M. Kolbeck, A. Garsuch, T. Bolin, M. Balasubramanian and L. F. Nazar, Sulfur Speciation in Li-S Batteries Determined by Operando X-Ray Absorption Spectroscopy, J. Phys. Chem. Lett., 2013, 4, 3227-3232.

11 S. Ma, L. Wang, Y. Wang, P. Zuo, M. He, H. Zhang, L. Ma, T. Wu and G. Yin, Palladium Nanocrystals-Imbedded Mesoporous Hollow Carbon Spheres with Enhanced Electrochemical Kinetics for High Performance Lithium Sulfur Batteries, Carbon, 2019, 143, 878-889.

12 F. Wu, J. Chen, R. Chen, S. Wu, L. Li, S. Chen and T. Zhao, Sulfur/Polythiophene with a Core/Shell Structure: Synthesis and Electrochemical Properties of the Cathode for Rechargeable Lithium Batteries, J. Phys. Chem. C, 2011, 115, 6057-6063.

13 Q. Pang, A. Shyamsunder, B. Narayanan, C. Y. Kwok, L. A. Curtiss and L. F. Nazar, Tuning the Electrolyte Network Structure to Invoke Quasi-Solid State Sulfur Conversion and Suppress Lithium Dendrite Formation in Li-S Batteries, Nat. Energy, 2018, 3, 783-791.

14 R. Dominko, A. Vizintin, G. Aquilanti, L. Stievano, M. J. Helen, A. R. Munnangi, M. Fichtner and I. Arcon, Polysulfides Formation in Different Electrolytes from the Perspective of X-Ray Absorption Spectroscopy, J. Electrochem. Soc., 2018, 165, A5014-A5019.

15 K. H. Wujcik, J. Velasco-Velez, C. H. Wu, T. Pascal, A. A. Teran, M. A. Marcus, J. Cabana, J. Guo, D. Prendergast and M. Salmeron, Fingerprinting Lithium-Sulfur Battery Reaction Products by X-Ray Absorption Spectroscopy, J. Electrochem. Soc., 2014, 161, A1100-A1106.

16 M. U. Patel, I. Arčon, G. Aquilanti, L. Stievano, G. Mali and R. Dominko, X-Ray Absorption near-Edge Structure and Nuclear Magnetic Resonance Study of the Lithium-Sulfur Battery and Its Components, ChemPhysChem, 2014, 15, 894-904.

17 G. Tan, R. Xu, Z. Xing, Y. Yuan, J. Lu, J. Wen, C. Liu, L. Ma, C. Zhan and Q. Liu, Burning Lithium in $\mathrm{Cs}_{2}$ for HighPerforming Compact $\mathrm{Li}_{2} \mathrm{~S}$-Graphene Nanocapsules for Li-S Batteries, Nat. Energy, 2017, 2, 17090.

18 Q. Guo, K. C. Lau and R. Pandey, Implication of Mechanical Properties of Li-S Binary Compounds Obtained from the First-Principles Study, J. Phys. Chem. C, 2021, 125, 290-294.

19 Z. Lin, C. Nan, Y. Ye, J. Guo, J. Zhu and E. J. Cairns, HighPerformance Lithium/Sulfur Cells with a Bi-Functionally Immobilized Sulfur Cathode, Nano Energy, 2014, 9, 408-416.

20 T. A. Pascal, K. H. Wujcik, J. Velasco-Velez, C. Wu, A. A. Teran, M. Kapilashrami, J. Cabana, J. Guo, M. Salmeron and N. Balsara, X-Ray Absorption Spectra of Dissolved Polysulfides in Lithium-Sulfur Batteries from First-Principles, J. Phys. Chem. Lett., 2014, 5, 1547-1551.
21 Y. Wang, J. Lv, L. Zhu and Y. Ma, Calypso: A Method for Crystal Structure Prediction, Comput. Phys. Commun., 2012, 183, 2063-2070.

22 Y. Wang, J. Lv, L. Zhu and Y. Ma, Crystal Structure Prediction Via Particle-Swarm Optimization, Phys. Rev. B: Condens. Matter Mater. Phys., 2010, 82, 094116.

23 G. Wang, R. Pandey and S. P. Karna, Carbon Phosphide Monolayers with Superior Carrier Mobility, Nanoscale, 2016, 8, 8819-8825.

24 G. Yang, S. Shi, J. Yang and Y. Ma, Insight into the Role of $\mathrm{Li}_{2} \mathrm{~S}_{2}$ in Li-S Batteries: A First-Principles Study, J. Mater. Chem. A, 2015, 3, 8865-8869.

25 J. Lv, Y. Wang, L. Zhu and Y. Ma, Predicted Novel HighPressure Phases of Lithium, Phys. Rev. Lett., 2011, 106, 015503.

26 G. Kresse and J. Furthmüller, Efficient Iterative Schemes for Ab Initio Total-Energy Calculations Using a Plane-Wave Basis Set, Phys. Rev. B: Condens. Matter Mater. Phys., 1996, 54, 11169-11186.

27 G. Kresse and J. Furthmüller, Efficiency of Ab-Initio Total Energy Calculations for Metals and Semiconductors Using a Plane-Wave Basis Set, Comput. Mater. Sci., 1996, 6, 15-50.

28 P. E. Blöchl, Projector Augmented-Wave Method, Phys. Rev. B: Condens. Matter Mater. Phys., 1994, 50, 17953.

29 J. P. Perdew, K. Burke and M. Ernzerhof, Generalized Gradient Approximation Made Simple, Phys. Rev. Lett., 1996, 77, 3865.

30 S. Grimme, Semiempirical Gga-Type Density Functional Constructed with a Long-Range Dispersion Correction, J. Comput. Chem., 2006, 27, 1787-1799.

31 H. Park, H. S. Koh and D. J. Siegel, First-Principles Study of Redox End Members in Lithium-Sulfur Batteries, J. Phys. Chem. C, 2015, 119, 4675-4683.

32 Q. Guo, K. C. Lau and R. Pandey, Thermodynamic and Mechanical Stability of Crystalline Phases of $\mathrm{Li}_{2} \mathrm{~S}_{2}, J$. Phys. Chem. C, 2019, 123, 4674-4681.

33 D. Prendergast and G. Galli, X-Ray Absorption Spectra of Water from First Principles Calculations, Phys. Rev. Lett., 2006, 96, 215502.

34 P. Giannozzi, S. Baroni, N. Bonini, M. Calandra, R. Car, C. Cavazzoni, D. Ceresoli, G. L. Chiarotti, M. Cococcioni and I. Dabo, Quantum Espresso: A Modular and Open-Source Software Project for Quantum Simulations of Materials, J. Phys.: Condens. Matter, 2009, 21, 395502.

35 P. Giannozzi, O. Andreussi, T. Brumme, O. Bunau, M. B. Nardelli, M. Calandra, R. Car, C. Cavazzoni, D. Ceresoli and M. Cococcioni, Advanced Capabilities for Materials Modelling with Quantum Espresso, J. Phys.: Condens. Matter, 2017, 29, 465901.

36 C. Gougoussis, M. Calandra, A. P. Seitsonen and F. Mauri, First-Principles Calculations of X-Ray Absorption in a Scheme Based on Ultrasoft Pseudopotentials: From A-Quartz to High$\mathrm{T}_{\mathrm{C}}$ Compounds, Phys. Rev. B: Condens. Matter Mater. Phys., 2009, 80, 075102.

37 M. Taillefumier, D. Cabaret, A.-M. Flank and F. Mauri, X-Ray Absorption near-Edge Structure Calculations with the 
Pseudopotentials: Application to the K Edge in Diamond and A-Quartz, Phys. Rev. B: Condens. Matter Mater. Phys., 2002, 66, 195107.

38 A. H. England, A. M. Duffin, C. P. Schwartz, J. S. Uejio, D. Prendergast and R. J. Saykally, On the Hydration and Hydrolysis of Carbon Dioxide, Chem. Phys. Lett., 2011, 514, 187-195.

39 T. A. Pascal, C. Pemmaraju and D. Prendergast, X-Ray Spectroscopy as a Probe for Lithium Polysulfide Radicals, Phys. Chem. Chem. Phys., 2015, 17, 7743-7753.

40 E. Rühl, R. Flesch, W. Tappe, D. Novikov and N. Kosugi, Sulfur 1s Excitation of $\mathrm{S}_{2}$ and $\mathrm{S}_{8}$ : Core-Valence-and ValenceValence-Exchange Interaction and Geometry-Specific Transitions, J. Chem. Phys., 2002, 116, 3316-3322.

41 Q. Guo, K. C. Lau and R. Pandey, Novel Metallic Crystalline Phase of $\mathrm{Li}_{2} \mathrm{~S}_{3}, J$. Phys. Chem. C, 2019, 123, 28027-28034.

42 Y. Li, N. A. Romero and K. C. Lau, Structure-Property of Lithium-Sulfur Nanoparticles Via Molecular Dynamics Simulation, ACS Appl. Mater. Interfaces, 2018, 10, 37575-37585.

43 W. Sun, S. T. Dacek, S. P. Ong, G. Hautier, A. Jain, W. D. Richards, A. C. Gamst, K. A. Persson and G. Ceder, The Thermodynamic Scale of Inorganic Crystalline Metastability, Sci. Adv., 2016, 2, e1600225.

44 M. J. Frisch, et al., Gaussian 16 Rev. C.01, Wallingford, CT, 2016.
45 J. Gao, M. A. Lowe, Y. Kiya and H. D. Abruna, Effects of Liquid Electrolytes on the Charge-Discharge Performance of Rechargeable Lithium/Sulfur Batteries: Electrochemical and in-Situ X-Ray Absorption Spectroscopic Studies, J. Phys. Chem. C, 2011, 115, 25132-25137.

46 Y. Gorlin, M. U. Patel, A. Freiberg, Q. He, M. Piana, M. Tromp and H. A. Gasteiger, Understanding the Charging Mechanism of Lithium-Sulfur Batteries Using Spatially Resolved Operando X-Ray Absorption Spectroscopy, J. Electrochem. Soc., 2016, 163, A930.

47 K. H. Wujcik, T. A. Pascal, C. Pemmaraju, D. Devaux, W. C. Stolte, N. P. Balsara and D. Prendergast, Characterization of Polysulfide Radicals Present in an Ether-Based Electrolyte of a Lithium-Sulfur Battery During Initial Discharge Using in Situ X-Ray Absorption Spectroscopy Experiments and First-Principles Calculations, Adv. Energy Mater., 2015, 5, 1500285.

48 Y. Zhang, R. S. Glass, K. Char and J. Pyun, Recent Advances in the Polymerization of Elemental Sulphur, Inverse Vulcanization and Methods to Obtain Functional Chalcogenide Hybrid Inorganic/Organic Polymers (Chips), Polym. Chem., 2019, 10, 4078-4105.

49 K. Sun, C. Zhao, C.-H. Lin, E. Stavitski, G. J. Williams, J. Bai, E. Dooryhee, K. Attenkofer, J. Thieme and Y.-c. K. ChenWiegart, Operando Multi-Modal Synchrotron Investigation for Structural and Chemical Evolution of Cupric Sulfide (Cus) Additive in Li-S Battery, Sci. Rep., 2017, 7, 1-10. 